\title{
Introduction to Mammalian Genome Special Issue: Genome Editing
}

\author{
Lydia Teboul $^{1} \cdot$ Yann Hérault $^{2,3,4,5,6} \cdot$ Cynthia Smith $^{7} \cdot$ Bruce Whitelaw $^{8}$
}

Published online: 15 July 2017

(C) Springer Science+Business Media, LLC 2017

Not quite 5 years on, the repurposing of the CRISPR system for genome engineering has fundamentally changed how we think about biomedical research and modelling. It has extended the range of genome manipulations that are possible, shrunk timelines as well as the cost of in vivo genome engineering, and has created many models for in vitro experimentation. We present a special issue of Mammalian Genome that takes stock of these advances and places them in the broad context of genomics.

As with all revolutions, although it seemed to take the field by surprise, the beginnings of these changes were noticeable with the use of entirely protein based genome editing tools, such as the meganucleases, Zinc-finger

Lydia Teboul

1.teboul@har.mrc.ac.uk

1 Mary Lyon Centre, MRC Harwell Institute, Harwell Science and Innovation Campus, Didcot OX11 0RD, UK

2 CELPHEDIA, PHENOMIN, Institut Clinique de la Souris (ICS), CNRS, INSERM, Université de Strasbourg, 1 rue Laurent Fries, 67404 Illkirch, France

3 Institut de Génétique et de Biologie Moléculaire et Cellulaire, Université de Strasbourg, 1 rue Laurent Fries, 67404 Illkirch, France

4 Centre National de la Recherche Scientifique, UMR7104 Illkirch, France

5 Institut National de la Santé et de la Recherche Médicale, U964 Illkirch, France

6 Université de Strasbourg, 1 rue Laurent Fries, 67404 Illkirch, France

7 Mouse Genome Informatics, The Jackson Laboratory, Bar Harbor 04609, USA

8 The Roslin Institute and Royal (Dick) School of Veterinary Studies, University of Edinburgh, Easter Bush, Midlothian, UK nucleases and transcription activator-like effector nucleases (TALENs). These initial advances provided new possibilities in terms of the type of genome modifications and the experimental models that realistically could be accessed. However, both the cost and intricacies of optimisation and implementation of these new tools restricted their broad adoption in research.

The introduction to the field of simple CRISPR-derived molecules for genome editing changed this. This issue brings together reviews that summarise the development of the genome engineering field (Fernandez et al.), describe the increasingly diverse toolset based on the CRISPR systems (Cebrian-Serrano and Davies) and highlight further advances to master genome editing outcome (Danner et al.). These tools allow the precise dissection of the function of specific sequences in the genome (as illustrated by Szafranski et al.). Also, new software to support the design and implementation of experiments plays a key role in facilitating the use of CRISPR tools in genome editing (as illustrated in this issue by Peterson et al.).

This issue contains reviews that describe how the new methods have led to the development of many novel experimental paradigms: rodents are modified to produce more complex models of human disease (Birling et al.) and other species have become readily accessible to genome engineering as summarised by Meek et al. (rat), Woodcock et al. (birds), Eaton and Wishart, and Telugu (larger animals). Importantly, the manipulation of the human genome is now also accessible in in vitro systems as presented here by Andrew Bassett.

But with novel methods and new experimental models comes the requirement to re-address classical questions: Wells and Joly discuss the issue of the classification of genome-edited models, Knowlton and Smith guide us through model nomenclature, Teboul et al. debate the 
validity of first generation genome-edited animals as models for phenotyping, Kenyon and Fray discuss the usefulness of biobanks in a time where genome editing seems so accessible and Andy Greenfield considers the ethical issues associated with the new technology.
The breadth of topics covered in this special issue reflects the broad impact on both fundamental and biomedical research of the recent developments in genome editing. 